\title{
Phase II trial of weekly nab-paclitaxel and carboplatin treatment with or without trastuzumab as nonanthracycline neoadjuvant chemotherapy for locally advanced breast cancer
}

\author{
This article was published in the following Dove Press journal: \\ International Journal of Nanomedicine \\ II March 2015 \\ Number of times this article has been viewed
}

\author{
Liang Huang 1,2 \\ Sheng Chen ${ }^{1,2}$ \\ Ling Yao ${ }^{1,2}$ \\ Guangyu Liu',2 \\ jiong $\mathrm{Wu}^{1,2}$ \\ Zhiming Shao ${ }^{1-3}$ \\ 'Department of Breast Surgery, Fudan \\ University Shanghai Cancer Center/ \\ Cancer Institute, ${ }^{2}$ Department of \\ Oncology, Shanghai Medical College, \\ ${ }^{3}$ Institutes of Biomedical Science, \\ Fudan University, Shanghai, People's \\ Republic of China
}

Background: Neoadjuvant chemotherapy has become standard treatment for women with locally advanced breast cancer. The aim of this study was to compare the efficacy and safety of nanoparticle albumin-bound paclitaxel (nab-paclitaxel) versus paclitaxel combined with carboplatin.

Methods: Thirty patients were treated with neoadjuvant nab-paclitaxel $\left(125 \mathrm{mg} / \mathrm{m}^{2}\right.$, days 1 , 8 , and 15) and carboplatin (area under the curve $=2$; days 1, 8, and 15) every 21 days for four cycles. Ninety matched patients received paclitaxel $\left(80 \mathrm{mg} / \mathrm{m}^{2}\right.$, days 1,8 , and 15$)$ and carboplatin every 21 days for four cycles. Weekly trastuzumab is recommended for overexpression of human epidermal receptor-2. The primary endpoint was pathologic complete response (defined as ypT0/is ypN0). Matching was conducted according to six variables: body mass index, clinical tumor stage, clinical lymph node status, estrogen receptor status, HER2 status, and trastuzumab receiving rate.

Results: Ninety percent of patients in the nab-paclitaxel group and $80 \%$ of patients in the paclitaxel group experienced a clinical objective response (complete response or partial response; $P=0.450$ ). Eight patients in the nab-paclitaxel group and 23 patients in the paclitaxel group had a pathologic complete response in the breast and axillary nodes $(26.7 \%$ versus $25.6 \%$; $P=0.904)$. Nab-paclitaxel showed a beneficial effective trend on clinical tumor stage II $(36.8 \%$ versus $15.8 \%$; $P=0.051$ ). When trastuzumab was added to nab-paclitaxel, the pathologic complete response rate was not significantly improved more than with trastuzumab and paclitaxel (43.6\% versus $39.6 \% ; P=0.769$ ). Carboplatin plus nab-paclitaxel or paclitaxel had similarly low pathologic complete response rates $(7.7 \%$ versus $10.5 \%)$ for the luminal molecular subtype. One (50\%) triple-negative patient achieved a pathologic complete response. The nab-paclitaxel regimen caused more grade 4 neutropenia than the paclitaxel regimen $(56.7 \%$ versus $21.1 \%$; $P<0.001)$.

Conclusion: Our study shows that weekly nab-paclitaxel and carboplatin with or without trastuzumab resulted in a pathologic complete response rate that was not superior to the matched cohorts. Future, larger trials are needed to validate that nab-paclitaxel is beneficial for clinical tumor stage II and the triple-negative subgroup.

Keywords: carboplatin, nanoparticle albumin-bound paclitaxel, neoadjuvant chemotherapy, pathologic complete response

\section{Introduction}

The survival of patients with early breast cancer has improved substantially in the past 40 years. ${ }^{1-3}$ The management of locally advanced breast cancer remains challenging, 
with high rates of locoregional and distant recurrences and significant morbidity and mortality. Neoadjuvant chemotherapy (NCT) has been a relatively standard treatment for locally advanced and initially inoperable breast cancer. This strategy also allows patients to undergo breast-conserving surgery and provides information on the efficacy of chemotherapy.

A pathologic complete response ( $\mathrm{pCR}$ ) has been the most commonly used endpoint in neoadjuvant trials, and the prognostic value is greatest in aggressive tumor subtypes, such as triple-negative, human epidermal receptor-2 (HER2)positive/hormone receptor-negative and luminal type with high risk. ${ }^{4}$ Some researchers believe that if novel agents produce a marked absolute increase in the frequency of a pCR compared with standard therapy alone in the intention-to-treat population, then those agents could also be reasonably likely to result in long-term improvements in event-free survival and overall survival. ${ }^{4}$

To minimize potentially severe hypersensitivity reactions and treatment-limiting toxicities, nab-paclitaxel has been developed by encapsulating hydrophobic paclitaxel molecules within human serum albumin particles. This formulation also enhances tumor uptake of paclitaxel by activating gp60, the endothelial cell surface receptor for albumin. ${ }^{5}$ In addition, nab-paclitaxel reached the tumor microenvironment more efficiently in preclinical models. ${ }^{6}$ In perverse comparative studies, nab-paclitaxel achieved a significantly higher overall response and longer time to progression than paclitaxel or docetaxel in metastatic breast cancer. ${ }^{7,8}$ However, in the neoadjuvant setting, only some small Phase II trials, containing a small number of patients, have discussed the value of nab-paclitaxel.

On the other hand, there is a lack of direct studies comparing the efficacy of nab-paclitaxel and solvent-based paclitaxel. In this matched study, we randomly selected treated controls, and patients treated with nab-paclitaxel, in a strict $3: 1$ ratio. We herein report the results that directly compare the efficacy and safety of nab-paclitaxel and solvent-based paclitaxel in a neoadjuvant setting.

\section{Patients and methods}

This Phase II trial of the neoadjuvants nab-paclitaxel and carboplatin was started in December 2011 and performed by the Shanghai Cancer Center Consortium. Trastuzumab was recommended for all patients with overexpression of HER2. The protocol was reviewed and approved by the independent ethics committee and institutional review board of Shanghai Cancer Center, Fudan University. All patients provided written informed consent before being included in this trial.
This nab-paclitaxel trial is registered at ClinicalTrials.gov (NCT01625429).

\section{Eligibility}

Women aged 18-70 years with histologically proven, corebiopsied, invasive (except inflammatory) breast cancer who were scheduled to receive preoperative chemotherapy were eligible. The participants were required to have clinical stage II or III breast cancer with a clinical or radiographically measurable residual tumor after core biopsy. Other key inclusion criteria included the following: assessable tumor in the breast without evidence of distant metastasis, as measured by bilateral breast mammogram, magnetic resonance imaging, chest computed tomography scan, abdominal ultrasound, and bone scan; Eastern Cooperative Oncology Group performance status 0 or 1; left ventricular ejection fraction $\geq 55 \%$, as measured by echocardiography; and not pregnant or lactating. Patients were required to have adequate hematopoietic function (absolute neutrophil count $\geq 1.5 \times 10^{9} / \mathrm{L}$, platelet count $\geq 100 \times 10^{9} / \mathrm{L}$, and hemoglobin level $\geq 100 \mathrm{~g} / \mathrm{L}$ ), adequate hepatic and renal function (bilirubin level, aspartate aminotransferase, and alanine aminotransferase $<1.5$ times the normal upper limits and serum creatinine $<110 \mu \mathrm{mol} / \mathrm{L})$. Patients were not eligible if they had concurrent uncontrolled underlying disease, distant metastases, inadequate bone marrow or renal function, impaired liver function, impaired cardiac function, or known serious allergy to any of the study drugs.

The matches were chosen from the eligible database according to six variables: body mass index, clinical tumor stage, clinical lymph node status, estrogen receptor (ER) status, HER2 status, and trastuzumab receiving rate. After matching, the controls were randomly selected in a strict $3: 1$ ratio to patients in the nab-paclitaxel group.

\section{Treatment}

In this matched study, patients received weekly treatment with nab-paclitaxel $\left(125 \mathrm{mg} / \mathrm{m}^{2}\right)$ or paclitaxel $\left(80 \mathrm{mg} / \mathrm{m}^{2}\right)$ for 30 minutes and carboplatin (area under the curve [AUC] 2) in each group as calculated using the Calvert formula. Patients with HER2 overexpression were recommended to receive weekly combined trastuzumab (initial dose $4 \mathrm{mg} / \mathrm{kg}$, with subsequent doses of $2 \mathrm{mg} / \mathrm{kg}$ ). The NCT was administered every 3 weeks for four cycles, and breast surgery with level I-II axillary dissection was performed 2-4 weeks after the last chemotherapy dose. The type of surgery was at the surgeon's discretion. Adjuvant chemotherapy began within 4 weeks postoperatively. An additional two cycles 
of paclitaxel and carboplatin treatment were delivered to patients who achieved a pCR; otherwise, four cycles of epirubicin and cyclophosphamide were administered. Trastuzumab administration for one year was recommended for patients with overexpression of HER2.

\section{Assessment}

Immunohistochemistry was performed on formalin-fixed, paraffin-embedded tissue sections from tumor specimens using standard procedures to evaluate biomarkers, including ER, the progesterone receptor (PR), HER-2, and Ki-67 before NCT. The cut-off value for ER and PR positivity was $1 \%$ of tumor cells with positive nuclear staining. Hormonal receptor positivity was defined as either ER-positive or PR-positive; however, a tumor that was hormone receptornegative was defined as both ER-negative and PR-negative. HER-2 was evaluated as $0,1+, 2+$, or $3+$ using circumferential, membrane-bound staining (HercepTest; Dako Cytomation, Carpinteria, CA, USA), and for $2+$ samples, fluorescent in situ hybridization was performed to detect the amplified gene. Samples that were $3+$ by immunohistochemistry or positive by fluorescent in situ hybridization were considered HER-2 positive. ${ }^{9}$ Positivity for Ki-67 was determined by nuclear staining in more than $14 \%$ of cells, with a minimum of 1,000 cells. The following antibodies were used for immunohistochemistry: ER (M7047, clone 1D5, Dako, Produktionsvej, Glostrup, Denmark), PR (M3569, clone PgR 636, Dako), HER-2 (A0485, polyclonal rabbit antibody, Dako) and Ki-67 (M7240, clone MIB-1, Dako). The immunohistochemical slides were evaluated independently by two pathologists. ${ }^{10}$

\section{Definition of response}

In this trial, pCR (ypT0/is ypN0) was defined as the absence of invasive cancer in the breast and axillary nodes, irrespective of ductal carcinoma in situ. ${ }^{4}$ Clinical tumor response was defined as a complete response if there was no clinical evidence of palpable tumor in either the breast or axilla at the time of surgery. Reduction of total tumor size by $\geq 30 \%$ at the time of surgery was considered a partial response. Progressive disease was noted if there was evidence of an increase $\geq 20 \%$ in the total calculated product of the tumor measurements or if a new lesion developed. Patients not meeting the criteria for either an objective response or progression were considered to have stable disease. Adverse events were graded according to the National Cancer Institute Common Terminology Criteria for Adverse Events, version 4.0.

\section{Statistical analysis}

Descriptive statistics were calculated to summarize the patient characteristics, tumor size, and biomarker levels in the core needle biopsies and surgical tumor samples. Toxicity rates in the two cohorts were presented using frequency tabulations with the corresponding percentages. The chisquared test was used to evaluate the relationship between patient characteristics and clinical response. A multivariate logistic regression model for predicting the response to NCT was used when combining a series of variables. The odds ratios (ORs) with $95 \%$ confidence interval (CIs) were calculated by logistic regression analysis for every variable. The efficacy and safety analyses were done according to the intention-to-treat principle, with all patients included according to their assignment. All statistical procedures were carried out using Statistical Package for the Social Sciences version 13.0 (SPSS Inc, Chicago, IL, USA) and Stata version 11.0 (StataCorp, College Station, TX, USA).

\section{Results \\ Patient characteristics}

From September 2010 to July 2013, 120 patients were enrolled at Fudan University, Shanghai Cancer Center. Of these patients, 30 were assigned to the nab-paclitaxel group and 90 were assigned to the matched paclitaxel group. The matches were chosen according to six variables: body mass index, clinical tumor stage, clinical lymph node status, ER status, HER2 status, and trastuzumab receiving rate. The patients' other disease characteristics were well balanced between the groups (Table 1). In the nab-paclitaxel group, the median age was 49 (range 29-66) years and 20 patients were premenopausal. In the matched group, the median age was 47.5 (range 24-71) years and 49 patients were premenopausal. In both groups, $56.7 \%$ of the patients had clinical tumor stage II tumors and $36.7 \%$ had stage III tumors. Seventy percent of the patients in both groups were clinically axillary lymph node-positive. The ER status was positive in $66.7 \%$ of patients in both the nab-paclitaxel and paclitaxel groups. The PR and Ki67 statuses were well balanced; the cut-off value for Ki67 was 14\%. One patient with HER2 overexpression in the nab-paclitaxel group refused treatment with trastuzumab. To balance the two groups, we also enrolled three patients who were HER2-positive and who received NCT without trastuzumab.

\section{Efficacy and responses}

Six patients in the nab-paclitaxel group and nine patients in the paclitaxel group did not complete the recommended four 
Table I Patient characteristics

\begin{tabular}{|c|c|c|c|}
\hline & $\begin{array}{l}\text { Nab-paclitaxel } \\
\text { cohort }\end{array}$ & $\begin{array}{l}\text { Paclitaxel } \\
\text { cohort }\end{array}$ & $P$-value \\
\hline Age, years & & & 0.285 \\
\hline$\geq 45$ & 20 & 50 & \\
\hline$<45$ & 10 & 40 & \\
\hline ECOG & & & 1.000 \\
\hline 0 & 29 & 88 & \\
\hline I & I & 2 & \\
\hline Menopause & & & 0.241 \\
\hline Yes & 10 & 41 & \\
\hline No & 20 & 49 & \\
\hline BMI & & & I \\
\hline$\geq 25$ & 8 & 24 & \\
\hline$<25$ & 22 & 66 & \\
\hline Clinical tumor stage & & & 1 \\
\hline I & 2 & 6 & \\
\hline 2 & 17 & 51 & \\
\hline 3 & 11 & 33 & \\
\hline Clinical nodal stage & & & l \\
\hline Positive & 21 & 63 & \\
\hline Negative & 9 & 27 & \\
\hline ER & & & I \\
\hline Positive & 20 & 60 & \\
\hline Negative & 10 & 30 & \\
\hline PR & & & 0.915 \\
\hline Positive & 17 & 52 & \\
\hline Negative & 13 & 38 & \\
\hline HER2 & & & l \\
\hline Positive & 17 & 51 & \\
\hline Negative & 13 & 39 & \\
\hline Ki67 & & & 0.482 \\
\hline$\geq 14 \%$ & 26 & 82 & \\
\hline$<14 \%$ & 4 & 8 & \\
\hline Target therapy & & & l \\
\hline Yes & 16 & 48 & \\
\hline No & 14 & 42 & \\
\hline
\end{tabular}

Abbreviations: BMI, body mass index; ECOG, Eastern Cooperative Oncology Group; ER, estrogen receptor; PR, progesterone receptor; HER2, human epidermal receptor-2.

cycles of chemotherapy because of an adverse event, disease progression, the patient's decision, or immediate surgery. When analyzing the group of patients who received treatment as planned, no difference was observed between the two groups. After completion of NCT, all patients received breast surgery. Overall, $90 \%$ of patients in the nab-paclitaxel group and $80 \%$ of patients in the paclitaxel group experienced a clinical objective response (complete response or partial response), as assessed by palpation, ultrasonography, and/ or magnetic resonance imaging $(P=0.450)$. The overall clinical response did not differ between the two cohorts (Table 2). Three patients in the nab-paclitaxel group and 14 patients in the paclitaxel group progressed or stabilized during treatment.
Table 2 Clinical and pathological responses

\begin{tabular}{llll}
\hline & $\begin{array}{l}\text { Nab-paclitaxel } \\
\text { cohort }\end{array}$ & $\begin{array}{l}\text { Paclitaxel } \\
\text { cohort }\end{array}$ & P-value \\
\hline $\begin{array}{l}\text { Finished cycles } \\
\text { Completed four cycles }\end{array}$ & 24 & 81 & 0.151 \\
$\begin{array}{l}\text { Did not complete four cycles } \\
\text { Clinical response }\end{array}$ & 6 & 9 & \\
CR/PR & 27 & 72 & 0.212 \\
SD/PD & 3 & 18 & \\
Breast pathological evaluation & 9 & & \\
PCR & 21 & 30 & \\
Non-pCR & 60.736 \\
Overall pathological evaluation & 60 & \\
PCR & 8 & & \\
Non-pCR & 22 & 23 & \\
\hline Abbrevition & 67 & \\
\hline
\end{tabular}

Abbreviations: $C R$, complete response; $P R$, partial response; $S D$, stable disease; $\mathrm{PD}$, progressive disease; $\mathrm{PCR}$, pathological complete response.

Eight (26.7\%) of 30 patients in the nab-paclitaxel group and $23(25.6 \%)$ of 90 patients in the paclitaxel group had a pCR in breast and lymph node (ypT0/is, ypN0; OR 1.059 95\% CI $0.415-2.705 ; P=0.904)$. When $\mathrm{pCR}$ was defined as the absence of invasive cancer only in the breast, this non-significant numerical difference was also noted $(30.0 \%$ versus 33.3\%; OR 0.857 95\% CI 0.35-2.099; $P=0.736$ ). In the subgroup analysis, we compared the efficacy of nab-paclitaxel and paclitaxel treatment in the breast and lymph node. No significant differences were found in age, menopause, body mass index, clinical tumor stage, clinical lymph node stage, ER status, PR status, HER2 status, Ki67 status, or target therapy (Figure 1). However, nab-paclitaxel tended to have a beneficial effect on clinical tumor stage II (36.8\% versus $15.8 \%$; OR $3.11195 \%$ CI $0.963-10.053$; $P=0.051)$.

\section{Safety and toxicity}

Table 3 shows the grade $1-4$ hematologic and nonhematologic adverse events that were observed during NCT. The most frequently occurring $3 / 4$ grade adverse events were hematology toxicity that only included neutropenia and leucopenia. The nab-paclitaxel regimen caused higher grade 4 neutropenia than the paclitaxel regimen (56.7\% versus $21.1 \%, P<0.001)$; no patient had febrile neutropenia in the nab-paclitaxel group and only one patient had febrile neutropenia in the paclitaxel group. Eight patients in the nab-paclitaxel group experienced asymptomatic grade 3 thrombocytopenia, which did not occur in the paclitaxel group. Nausea and vomiting were the most frequent nonhematologic toxic effects, but most cases were mild or moderate. Severe nonhematologic toxicity was relatively uncommon during neoadjuvant therapy in both groups, and only five grade 3 events (rash, nausea, vomiting, 


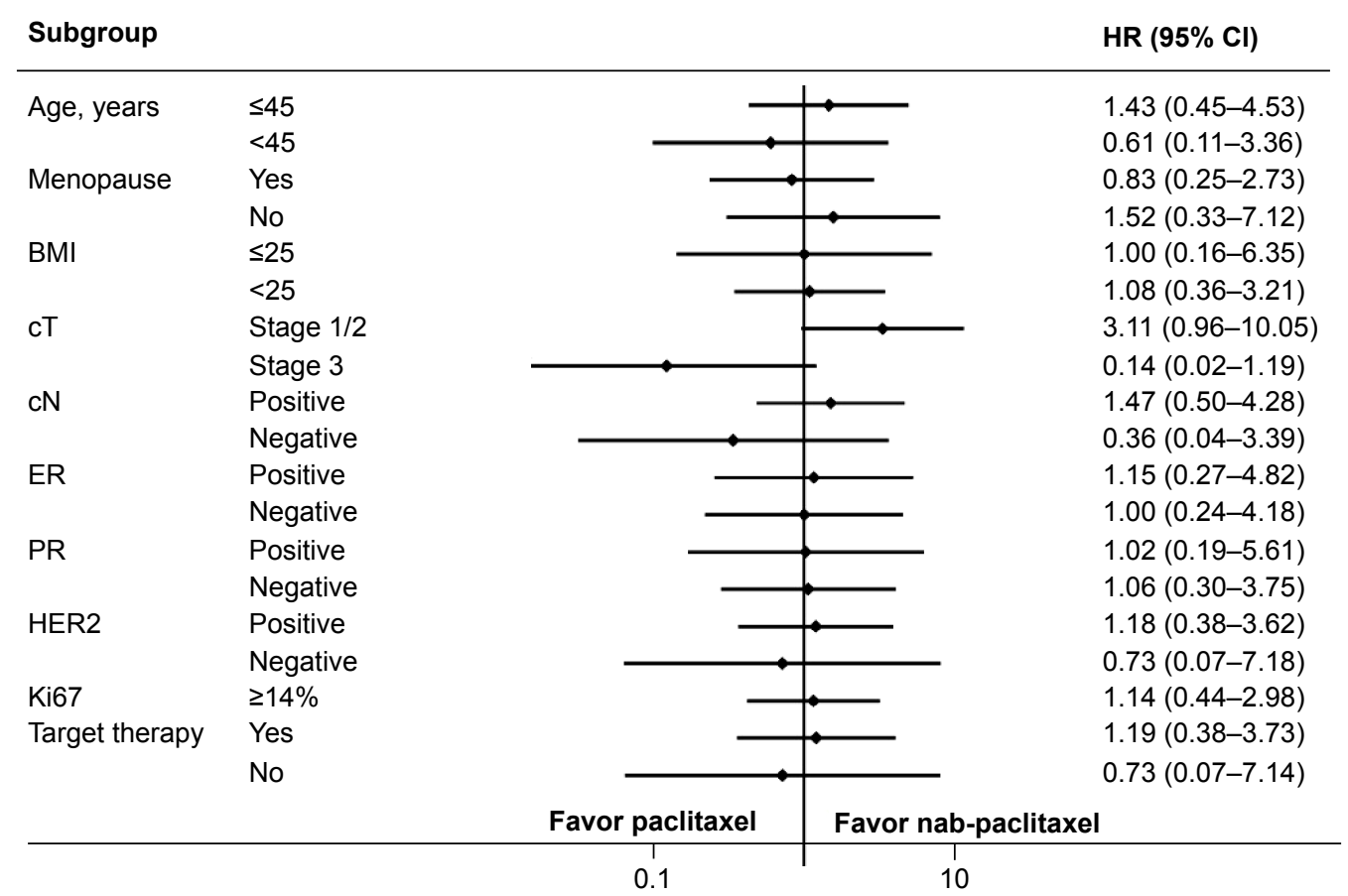

Figure I Comparison of the efficacy of nab-paclitaxel and paclitaxel in subgroup analysis.

Abbreviations: BMI, body mass index; $\mathrm{Cl}$, confidence interval; ER, estrogen receptor; HR, hazard ratio; PR, progesterone receptor; HER2, human epidermal receptor-2; cT, clinical tumor stage; cN, clinical lymph nodal stage.

and elevated liver enzymes) were observed. Mild palpitations were observed in two patients in the nab-paclitaxel group and in 12 patients in the paclitaxel group. Symptomatic congestive cardiac failure was not observed, and no difference was observed in the two cohorts. No serious adverse event occurred in the nab-paclitaxel group, and no treatment-related deaths occurred.

\section{Discussion}

Patients who attain a pCR (defined as ypT0 ypN0 or ypT0/ is ypN0) have improved survival, and the prognostic value

Table 3 Common treatment-related grade I-4 adverse events

\begin{tabular}{|c|c|c|c|c|c|c|c|c|}
\hline \multirow[t]{2}{*}{$\begin{array}{l}\text { Adverse event } \\
\text { (grade) }\end{array}$} & \multicolumn{4}{|c|}{$\begin{array}{l}\text { Nab-paclitaxel } \\
\text { cohort }\end{array}$} & \multicolumn{4}{|c|}{ Paclitaxel cohort } \\
\hline & $\mathbf{I}$ & 2 & 3 & 4 & $\mathbf{I}$ & 2 & 3 & 4 \\
\hline Anemia & 9 & 14 & 5 & 0 & 59 & 7 & 3 & 0 \\
\hline Thrombocyto & 3 & 7 & 8 & 0 & 10 & 5 & 0 & 0 \\
\hline Leukopenia & 0 & 6 & 21 & 2 & 10 & 43 & 35 & 0 \\
\hline Neutropenia & 0 & 0 & 13 & 17 & 10 & 26 & 33 & 19 \\
\hline Diarrhea/ & 10 & 8 & 0 & 0 & 42 & II & 2 & 0 \\
\hline Cardia & 2 & 0 & 0 & 0 & 11 & I & 0 & 0 \\
\hline Rash & 7 & 3 & 1 & 0 & 22 & I & 0 & 0 \\
\hline Hand-foot syndrome & 4 & 0 & 0 & 0 & 19 & 2 & 0 & 0 \\
\hline Nausea/vomiting & 15 & 13 & 2 & 0 & 72 & 9 & 1 & 0 \\
\hline Peripheral neurotoxicity & 13 & 0 & 0 & 0 & 28 & 3 & 0 & 0 \\
\hline Increased liver enzyme & 12 & 3 & 0 & 0 & 39 & 2 & I & 0 \\
\hline Increased bilirubin & 3 & 0 & 0 & 0 & 6 & 0 & 0 & 0 \\
\hline
\end{tabular}

is greatest in aggressive tumor subtypes. In our two-cohort group, most of the patients belonged to the aggressive molecular subgroup. Additional active agents, intensification of therapy of brief duration, and sequencing chemotherapy were strategies for improving the efficacy of neoadjuvant therapy. ${ }^{11-13}$

The primary aim of our study was to investigate the efficacy and safety of nab-paclitaxel combined with carboplatin treatment with or without trastuzumab. Although definitive conclusions cannot be drawn due to the small number of patients in these studies and due to the lack of confirmatory Phase III data, nab-paclitaxel monotherapy is being increasingly adopted to treat metastatic breast cancer after failure of anthracycline-based chemotherapy. Weekly nab-paclitaxel treatment was shown to have a superior therapeutic index compared with nab-paclitaxel and docetaxel every 3 weeks in some clinical trials. ${ }^{7,8}$

Avoidance of anthracyclines has reduced the potential for cardiotoxicity, and multiple clinical trials have demonstrated the efficacy of taxane/platinum combinations, even with trastuzumab. ${ }^{14-16}$ Results of a randomized Phase III trial in the adjuvant setting that compared doxorubicine-cyclophosphamide followed by docetaxel-trastuzumab with upfront docetaxel-carboplatin-trastuzumab showed equal efficacy for both treatment arms, but it showed less cardiotoxicity with the nonanthracycline regimen. ${ }^{17}$ For locally advanced breast 
cancer, there was very limited clinical evidence for weekly nab-paclitaxel and platinum as a neoadjuvant regimen. In the nab-paclitaxel cohort, the relatively high rate of treatment discontinuation was unusual $(10 \%$ patients did not complete four cycles). In our study, patients receiving nab-paclitaxel, carboplatin, and trastuzumab achieved a $43.6 \%$ pCR rate, which was similar to the $54 \%$ rate achieved in a Phase II pilot study using a combination of nab-paclitaxel, carboplatin, trastuzumab, and bevacizumab. ${ }^{18}$ In the BrUOG BR-211B trial, ten patients $(50 \%)$ treated with carboplatin every 3 weeks and weekly nab-paclitaxel and trastuzumab for 18 weeks were evaluated for pCR. ${ }^{19}$ Weekly nab-paclitaxel and carboplatin and biweekly bevacizumab resulted in six patients (18\%) achieving a pCR.$^{20}$ Our analysis confirmed this finding, indicating that nab-paclitaxel does not improve the $\mathrm{pCR}$ rate in luminal types of breast cancer.

Comparing the results of NCT studies is difficult because of differences in the duration of treatment, patient populations, and definitions of pCR. We selected six widely recognized predictive factors for patient matching including body mass index, clinical tumor stage, clinical lymph node status, ER status, HER2 status, and trastuzumab receiving rate. In the control cohort, 19 patients $(39.6 \%)$ had a pCR in the breast and lymph nodes after paclitaxel, carboplatin, and trastuzumab, which was within the previously reported range for various chemotherapy and target agent combinations. ${ }^{21}$ In agreement with our findings, a Phase II study of NCT in 107 patients showed that four cycles of weekly paclitaxel and carboplatin achieved a pCR rate of $19.4 \% .{ }^{22} \mathrm{Nab}$-paclitaxel tended to be beneficial for treating clinical tumor stage II $(P=0.051)$, but other small neoadjuvant clinical trials lack evidence to support this trend, and larger trials with subgroup analysis are needed for validation. In the triple-negative breast cancer subtype, the high pCR rate was confirmed by subgroup analysis of another clinical trial, even though only one patient (50\%) achieved a pCR in this trial. ${ }^{20} \mathrm{~A}$ regimen containing nab-paclitaxel might be effective in the subpopulation of patients with triple-negative breast cancer.

The limitations of this study should be acknowledged. First, although the sample size for solvent-paclitaxel is sufficient for analysis, that for nab-paclitaxel was underpowered for the comparative analysis. The findings require further validation. Second, because of the short-term follow-up duration, survival analysis is unavailable. Therefore, it is unclear whether the improvement in pCR would transfer into a survival benefit.

In this matched study, we demonstrated that the combination of nab-paclitaxel and a target agent had a high level of efficacy, but superiority of this combination to solventbased paclitaxel was not evident. On the other hand, the nab-paclitaxel/carboplatin and solvent-based paclitaxel/ carboplatin groups had similar pCR rates. Paclitaxel or nab-paclitaxel combined with carboplatin could cause a high rate of hematologic toxicity, as the most commonly reported grade $3 / 4$ toxicities, such as neutropenia, were over $70 \% .^{20,21}$ The grade $3 / 4$ hematologic adverse events, which are neoadjuvant complications, are worrisome and require careful evaluation in larger studies. The pCR rate in the nabpaclitaxel cohort was similar to that in the matched solventbased paclitaxel cohort; therefore, the role of nab-paclitaxel remains undefined. A trend suggesting that nab-paclitaxel was beneficial for clinical tumor stage II and triple-negative breast cancer was observed. Several large Phase III trials evaluating the effect of nab-paclitaxel in the neoadjuvant setting are currently ongoing and should provide definitive answers in the near future.

\section{Acknowledgments}

We are grateful to the patients who participated in this trial. This research was supported by Celgene and the National Natural Science Foundation of China (81302298). The funders had no role in the study design, data collection and analysis, decision to publish, or preparation of the manuscript.

\section{Disclosure}

The authors report no conflicts of interest in this work.

\section{References}

1. Autier P, Boniol M, La Vecchia C, et al. Disparities in breast cancer mortality trends between 30 European countries: retrospective trend analysis of WHO mortality database. BMJ. 2010;341:c3620.

2. Berry DA, Cronin KA, Plevritis SK, et al. Effect of screening and adjuvant therapy on mortality from breast cancer. $N$ Engl J Med. 2005; 353(17):1784-1792.

3. Edge SB. Advances in breast surgery, 2002-2012. J Natl Compr Canc Netw. 2013;11(1):53-59.

4. Cortazar P, Zhang L, Untch M, et al. Pathological complete response and long-term clinical benefit in breast cancer: the CTNeoBC pooled analysis. Lancet. 2014;384(9938):164-172.

5. Overmoyer B. Options for the treatment of patients with taxanerefractory metastatic breast cancer. Clin Breast Cancer. 2008;8 Suppl 2: S61-S70.

6. Desai N, Trieu V, Yao Z, et al. Increased antitumor activity, intratumor paclitaxel concentrations, and endothelial cell transport of cremophorfree, albumin-bound paclitaxel, ABI-007, compared with cremophorbased paclitaxel. Clin Cancer Res. 2006;12(4):1317-1324.

7. Gradishar WJ, Tjulandin S, Davidson N, et al. Phase III trial of nanoparticle albumin-bound paclitaxel compared with polyethylated castor oil-based paclitaxel in women with breast cancer. J Clin Oncol. 2005;23(31):7794-7803.

8. Gradishar WJ, Krasnojon D, Cheporov S, et al. Significantly longer progression-free survival with nab-paclitaxel compared with docetaxel as first-line therapy for metastatic breast cancer. J Clin Oncol. 2009;27(22):3611-3619. 
9. Dowsett M, Procter M, McCaskill-Stevens W, et al. Disease-free survival according to degree of HER2 amplification for patients treated with adjuvant chemotherapy with or without 1 year of trastuzumab: the HERA Trial. J Clin Oncol. 2009;27(18):2962-2969.

10. Huang L, Chen T, Chen C, et al. Prognostic and predictive value of Phospho-p44/42 and pAKT in HER2-positive locally advanced breast cancer patients treated with anthracycline-based neoadjuvant chemotherapy. World J Surg Oncol. 2013;11:307.

11. Earl HM, Vallier AL, Hiller L, et al. Effects of the addition of gemcitabine, and paclitaxel-first sequencing, in neoadjuvant sequential epirubicin, cyclophosphamide, and paclitaxel for women with high-risk early breast cancer (Neo-tAnGo): an open-label, $2 \times 2$ factorial randomised phase 3 trial. Lancet Oncol. 2014;15(2):201-212.

12. Yu KD, Liu GY, Chen CM, et al. Weekly paclitaxel/carboplatin/ trastuzumab therapy improves pathologic complete remission in aggressive HER2-positive breast cancers, especially in luminal-B subtype, compared with a once-every-3-weeks schedule. Oncologist. 2013;18(5):511-517.

13. Gianni L, Pienkowski T, Im YH, et al. Efficacy and safety of neoadjuvant pertuzumab and trastuzumab in women with locally advanced, inflammatory, or early HER2-positive breast cancer (NeoSphere): a randomised multicentre, open-label, phase 2 trial. Lancet Oncol. 2012;13(1):25-32.

14. Robert N, Leyland-Jones B, Asmar L, et al. Randomized phase III study of trastuzumab, paclitaxel, and carboplatin compared with trastuzumab and paclitaxel in women with HER-2-overexpressing metastatic breast cancer. J Clin Oncol. 2006;24(18):2786-2792.

15. Burris H 3rd, Yardley D, Jones S, et al. Phase II trial of trastuzumab followed by weekly paclitaxel/carboplatin as first-line treatment for patients with metastatic breast cancer. J Clin Oncol. 2004;22(9):1621-1629.
16. Bullock K, Blackwell K. Clinical efficacy of taxane-trastuzumab combination regimens for HER-2-positive metastatic breast cancer. Oncologist. 2008;13(5):515-525.

17. Slamon D, Eiermann W, Robert N, et al. Adjuvant trastuzumab in HER2positive breast cancer. $N$ Engl J Med. 2011;365(14):1273-1283.

18. Yardley DA, Raefsky E, Castillo R, et al. Phase II study of neoadjuvant weekly nab-paclitaxel and carboplatin, with bevacizumab and trastuzumab, as treatment for women with locally advanced HER2+ breast cancer. Clin Breast Cancer. 2011;11(5):297-305.

19. Cheng H, Bai Y, Sikov W, et al. Quantitative measurements of HER2 and phospho-HER2 expression: correlation with pathologic response to neoadjuvant chemotherapy and trastuzumab. BMC Cancer. 2014; $14: 326$.

20. Mrozek E, Layman R, Ramaswamy B, et al. Phase II trial of neoadjuvant weekly nanoparticle albumin-bound paclitaxel, carboplatin, and biweekly bevacizumab therapy in women with clinical stage II or III HER2-negative breast cancer. Clin Breast Cancer. 2014;14(4):228-234.

21. Sonke GS, Mandjes IA, Holtkamp MJ, et al. Paclitaxel, carboplatin, and trastuzumab in a neo-adjuvant regimen for HER2-positive breast cancer. Breast J. 2013;19(4):419-426.

22. Chen XS, Nie XQ, Chen CM, et al. Weekly paclitaxel plus carboplatin is an effective nonanthracycline-containing regimen as neoadjuvant chemotherapy for breast cancer. Ann Oncol. 2010;21(5):961-967.
International Journal of Nanomedicine

\section{Publish your work in this journal}

The International Journal of Nanomedicine is an international, peerreviewed journal focusing on the application of nanotechnology in diagnostics, therapeutics, and drug delivery systems throughout the biomedical field. This journal is indexed on PubMed Central, MedLine, CAS, SciSearch $®$, Current Contents $\AA /$ Clinical Medicine,

\section{Dovepress}

Journal Citation Reports/Science Edition, EMBase, Scopus and the Elsevier Bibliographic databases. The manuscript management system is completely online and includes a very quick and fair peer-review system, which is all easy to use. Visit http://www.dovepress.com/ testimonials.php to read real quotes from published authors. 